\title{
Study on Genotyping Polymorphism and Sequencing of N-Acetyltransferase 2 (NAT2) among Al-Ahsa Population
}

\author{
Mohammad Abu Zahra, ${ }^{1}$ Mahmoud Kandeel, ${ }^{1,2}$ Sara A. Aldossary, ${ }^{3}$ and Abdulla Al-Taher $\mathbb{D D}^{1}$ \\ ${ }^{1}$ Department of Biomedical Sciences, College of Veterinary Medicine, King Faisal University, 31982 Al-Ahsa, Saudi Arabia \\ ${ }^{2}$ Department of Pharmacology, Faculty of Veterinary Medicine, Kafrelsheikh University, 33516 Kafrelsheikh, Egypt \\ ${ }^{3}$ Department of Pharmaceutical Sciences, College of Clinical Pharmacy, King Faisal University, 31982 Al-Ahsa, Saudi Arabia
}

Correspondence should be addressed to Abdulla Al-Taher; aaltaher@kfu.edu.sa

Received 7 February 2020; Revised 4 May 2020; Accepted 12 May 2020; Published 15 June 2020

Academic Editor: Valeria Cavalcanti Rolla

Copyright ( ) 2020 Mohammad Abu Zahra et al. This is an open access article distributed under the Creative Commons Attribution License, which permits unrestricted use, distribution, and reproduction in any medium, provided the original work is properly cited.

\begin{abstract}
One of the well-studied phase II drug metabolizing enzymes is N-acetyltransferase 2 (NAT2) which has an essential role in the detoxification and metabolism of several environmental toxicants and many therapeutic drugs like isoniazid (antituberculosis, TB) and antimicrobial sulfonamides. According to the variability in the acetylation rate among different ethnic groups, individuals could be classified into slow, intermediate, and fast acetylators; these variabilities in the acetylation rate are a result of single nucleotide polymorphisms (SNPs) in the coding sequence of NAT2. The variety of NAT2 acetylation status is associated with some diseases such as bladder cancer, colorectal cancer, rheumatoid arthritis, and diabetes mellitus. The main objectives of this research are to describe the genetic profile of NAT2 gene among the people of the Al-Ahsa region, to detect the significant SNPs of this gene, to determine the frequency of major NAT2 alleles and genotypes, and then categorize them into fast, intermediate, and slow acetylators. Blood samples were randomly collected from 96 unrelated people from Al-Ahsa population, followed by DNA extraction then amplifying the NAT2 gene by polymerase chain reaction (PCR); finally, functional NAT2 gene (exon 2) was sequenced using the Sanger sequencing method. The well-known seven genetic variants of NAT2 gene are $191 \mathrm{G}>\mathrm{A}, 282 \mathrm{C}>\mathrm{T}, 341 \mathrm{~T}>\mathrm{C}, 481 \mathrm{C}>\mathrm{T}, 590 \mathrm{G}>\mathrm{A}, 803 \mathrm{~A}>\mathrm{G}$, and $857 \mathrm{G}>\mathrm{A}$ were detected with allele frequencies $1 \%, 35.4 \%$, $42.7 \%, 41.1 \%, 29.2 \%, 51 \%$, and $5.7 \%$, respectively. The most common NAT2 genetic variant among Al-Ahsa population was $803 \mathrm{~A}>\mathrm{G}$ with a high frequency 0.510 (95\% confidence interval $0.44-0.581$ ) followed by $341 \mathrm{~T}>\mathrm{C} 0.427$ (95\% confidence interval $0.357-0.497)$. The most frequent two haplotypes of $N A T 2$ were $N A T 2 * 6 C(25.00 \%)$ and $N A T 2 * 5 A(22.92 \%)$ which were classified as a slow acetylators. According to trimodal distribution of acetylation activity, the predicted phenotype of Al-Ahsa population was found to be $5.21 \%$ rapid acetylators, $34.38 \%$ intermediate acetylators, and $60.42 \%$ were slow acetylators. In addition, this study found four novel haplotypes $N A T 2 * 5 \mathrm{~TB}, N A T 2 * 5 A B, N A T 2 * 5 Z A$, and $N A T 2 * 6 \mathrm{~W}$ which were slow acetylators. This study revealed a high frequency of the NAT2 gene with slow acetylators $(60.42 \%)$ in Al-Ahsa population, which might alter the drug's efficacy and vulnerability to some diseases.
\end{abstract}

\section{Introduction}

Human NAT2 gene has a key role in the metabolism of hydrazines, arylamines, several environmental toxicants, and therapeutic drugs like isoniazid and the antimicrobial sulfonamides by the $\mathrm{N}$-acetylation of aromatic amines as well as the $\mathrm{O}$-acetylation of carcinogenic heterocyclic amines [1]. This gene is present on the chromosome number 8 , at Cytoband p22, and has a coding region of exon 2 with $870 \mathrm{bp}$ which encodes 290 amino acids, and it is mainly expressed in the liver and small intestine [2]. The interest of studying the variability in $\mathrm{N}$-acetylation status of NAT2 has been increased among ethnic groups due to the importance of NAT2 polymorphism as biomarkers to evaluate the efficacy of therapy and toxicity during treatment or minimization of adverse drug reactions (ADR) [3].

There is a relationship between NAT2 acetylation status with some diseases like bladder cancer, colorectal cancer, 
rheumatoid arthritis, and diabetes [3]. According to the variability in the acetylation rate among different ethnic groups, individuals are classified based on acetylation phenotypes as slow, intermediate, and rapid acetylators. This variability in the acetylation rate is a result of SNPs in the coding sequence of NAT2 [4]. The plasma drug concentration in slow acetylators remains higher than that in the rapid acetylators which may be correlated with adverse drug reactions (ADR) [5]. The most commonly found seven SNPs are $N A T 2 * 5$, $N A T 2 * 6, N A T 2 * 7, N A T 2 * 14, N A T 2 * 11, N A T 2 * 12$, and $N A T 2 * 13$ [6]. The wild-type NAT2*4 allele does not have any nucleotide substitutions and is associated with fast acetylator phenotype [7].

Ample literature studies show interethnic variation in acetylation. In addition, many studies showed a great variation between Arabs and non-Arabs in drugs and environmental chemical acetylation. Arabs, like others, are not a single race, and possible variation in drug metabolism is still existing. This could be due to the presence of different SNPs. Al-Ahsa is one of the most populated oasis since ancient times in the Arabian peninsula. Several ethnic groups migrated and settled at Al-Ahsa and merged to be the present inhabitants of Al-Ahsa. This may have made Al-Ahsa characterized by a mixed population structure, genetic makeup, and a host of special diseases such as sickle cell anemia. Because of the above, Al-Ahsa may be a promising place to study the genetic makeup of drug metabolizing enzymes and its role in drug response. Since there was no previous study of NAT2 (SNPs) in Al-Ahsa, so, this study was proposed. Hence, the present research is aimed at sequencing the NAT2 gene and identifying the gene polymorphisms, which may regulate the interethnic and interindividual phenotypes in drug's activity and toxicity.

\section{Materials and Methods}

2.1. Sample Collection. A total of 96 blood samples were randomly collected from unrelated individuals from different dispensaries of Al-Ahsa, Saudi Arabia. A volume of $3 \mathrm{ml}$ of venous blood samples were drawn and collected in EDTA tubes. Blood samples were placed in ice bags and then transported to the King Faisal University (KFU) laboratory where they were refrigerated at $4\left({ }^{\circ} \mathrm{C}\right)$ for DNA extraction.

Volunteers have been informed about the nature and the aim of the study, and they were asked to sign a consent before collection of samples. This work was approved by the Research Ethics Committee (REC) at King Faisal University (REC REF Number: KFU-REC/2017-11-02) and clinical ethical committee at King Fahad Hospital, Hufof (KFHH RCA NO: 07/09/39).

2.2. DNA Extraction and Quantification. The Wizard ${ }^{\circledR}$ Genomic DNA purification kit (Promega, Madison, USA) was used for extraction of genomic DNA from the white blood cells in each sample. The extracted gDNA is quantified by using a Nanodrop Spectrophotometer instrument (Synergy ${ }^{\mathrm{TM}}$ Mx Monochromator-Based Multi-Mode Microplate Reader, Bio-Tek, USA) and makes up the final concentration of $100 \mathrm{ng} / \mu \mathrm{l}$ by nuclease-free water.
2.3. Primer Design and NAT2 Gene Amplification by Polymerase Chain Reaction (PCR). The amplification of the NAT2 gene (exon 2) was carried out with a thermal cycler (C1000, Bio-Rad, Singapore). The PCR primers were designed according to the NAT2 sequence obtained from the National Center For Biotechnological Information (NCBI) and purchased from Macrogen, Korea. The NAT2specific primers were designed to amplify the exon 2 region of the NAT2 gene, forward primer $5^{\prime}$ GGGATCATGGA CATTGAAGCA3 ${ }^{\prime}$ and reverse primer $5^{\prime}$ ATGTTTTCTAG CATGAATCACTCTG (Macrogen, Seoul, Korea). The covering of the functional part of exon $2(870 \mathrm{bp})$ and its boundaries has a total length of $1150 \mathrm{bp}$. The PCR mixture consists of $100 \mathrm{ng}$ of genomic DNA, 2X Mastermix (2X Green GoTaq $^{\circledR}$ Reaction Buffer (pH 8.5), $400 \mu \mathrm{M}$ dATP, $400 \mu \mathrm{M}$ dGTP, $400 \mu \mathrm{M}$ dCTP, $400 \mu \mathrm{M}$ dTTP, and $3 \mathrm{mM} \mathrm{MgCl} 2$ ), and 10 picomoles of forward and reverse primers to make up the final reaction volume $50 \mu \mathrm{l}$. The PCR program was set as initial denaturation at $95^{\circ} \mathrm{C}$ for 3 minutes, 35 cycles were performed consisting of a denaturation step at $95^{\circ} \mathrm{C}$ for 30 seconds, an annealing step at $60^{\circ} \mathrm{C}$ for 30 seconds, and an elongation step at $72^{\circ} \mathrm{C}$ for 1 minute, completed with a final cycle of elongation at $72^{\circ} \mathrm{C}$ for 5 minutes.

2.4. NAT2 DNA Sequencing. The $1150 \mathrm{bp}$ amplicons of the NAT2 genes were sent to Macrogen company (Macrogen, Seoul, Korea) for sequencing. The detection of NAT2 variations was carried out by using a bidirectional highthroughput capillary sequencing based on Sanger's dideoxy chain-termination DNA sequencing method.

2.5. Sequence Data Analyses. The raw sequencing data were analyzed using a Mutation Surveyor ${ }^{\circledR}$ program (Pennsylvania, USA). It is a powerful, sensitive software and an accurate DNA sequencing analysis tool for Sanger sequencing files produced by Applied Biosystems Genetic Analyzers. For alignment, comparison, and screening for different SNPs, the variations of sample DNA sequence traces are compared to reference wild-type sequence from GenBank database. Each sequence was submitted to the program and compared with the NAT2 reference sequence. Moreover, the sequences of samples were evaluated for chromatogram peaks and overlapping bases to make consensus sequences. The homozygous and heterozygous SNPs cannot be differentiated by the software, so it has to look for each DNA sequence by naked eyes. Depending on the peak of the chromatogram, if there is a single clear and normal peak, this reflects that we have homozygous SNP, but if the peak was short with the presence of double peaks instead of one peak with different colors, that is an indication for heterozygous SNP.

2.6. Acetylator Phenotype Classification. Based on the level of NAT2 enzymatic activity, populations are divided into three NAT2 acetylator phenotypes: slow, intermediate, and rapid [4]. Hence, the predicted phenotypes in the current study were predicted from genotypes as three types of acetylators. A slow acetylator was predicted if the genotype was comprised of 2 slow alleles, a rapid acetylator genotype would consist of 2 rapid alleles, and an intermediate acetylator 


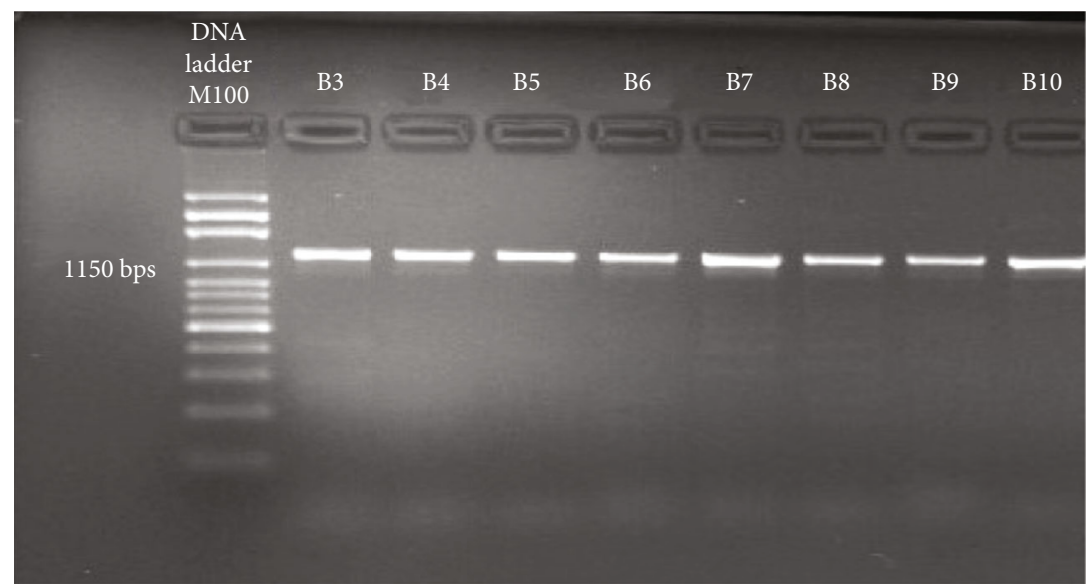

FIgURE 1: The PCR gel picture representing the $1150 \mathrm{bp}$ of NAT2 amplified fragment.

TABLE 1: The allele frequency of NAT2 polymorphisms among Al-Ahsa population $(n=96)$.

\begin{tabular}{|c|c|c|c|c|c|}
\hline NAT2 allele & Mutation site & Amino acid change & Reference ID & Acetylation status & $\begin{array}{l}\text { Allele frequency } \\
\qquad(95 \% \text { CI })\end{array}$ \\
\hline$N A T 2^{*} 4$ & - & Wild & - & Fast & $0.295(0.232-0.362)$ \\
\hline NAT2* 11 & $481 \mathrm{C}>\mathrm{T}$ & Leu161Leu & rs1799929 & Fast & $0.411(0.342-0.481)$ \\
\hline$N A T 2^{*} 12$ & $803 A>G$ & Arg268Lys & rs1208 & Fast & $0.510(0.44-0.581)$ \\
\hline$N A T 2^{*} 13$ & $282 \mathrm{C}>\mathrm{T}$ & Tyr94Tyr & rs1041983 & Fast & $0.354(0.287-0.422)$ \\
\hline$N A T 2 * 5$ & $341 \mathrm{~T}>\mathrm{C}$ & Ile114Thr & rs1801280 & Slow & $0.427(0.357-0.497)$ \\
\hline$N A T 2^{*} 6$ & $590 \mathrm{G}>\mathrm{A}$ & Arg197Gln & rs1799930 & Slow & $0.292(0.227-0.356)$ \\
\hline$N A T 2^{*} 7$ & $857 \mathrm{G}>\mathrm{A}$ & Gly286Glu & rs1799931 & Slow & $0.057(0.024-0.09)$ \\
\hline NAT2* 14 & $191 \mathrm{G}>\mathrm{A}$ & Arg64Gln & rs1801279 & Slow & $0.010(-0.0039-0.025)$ \\
\hline
\end{tabular}

would be predicted if its genotype contained one slow and another rapid acetylator allele. The alleles considered rapid were the wild $(N A T 2 * 4)$ ones and those containing polymorphisms of $282 \mathrm{C}>\mathrm{T}(N A T 2 * 13), 481 \mathrm{C}>\mathrm{T}(N A T 2 * 11)$, and $803 \mathrm{~A}>\mathrm{G} \quad(N A T 2 * 12)$, while all other alleles $341 \mathrm{~T}>\mathrm{C}$ $(N A T 2 * 5), 590 \mathrm{G}>\mathrm{A}(\mathrm{NAT} 2 * 6), 857 \mathrm{G}>\mathrm{A}(\mathrm{NAT} 2 * 7)$, and $191 \mathrm{G}>\mathrm{A}(N A T 2 * 14)$ were considered slow acetylators [8]. Online software NAT2PRED program (http://nat2pred.rit .albany.edu/) was used for inferring the human NAT2 acetylator phenotype that uses a combination of SNPs found in the NAT2 gene positions 282, 341, 481, 590, 803, and 857 [9].

2.7. Statistical Analysis. The chi-squared $\left(\chi^{2}\right)$ test was performed on observed and expected genotype frequencies to find out whether the genotype distribution was in HardyWeinberg equilibrium and to compare frequencies of genotypes and alleles in the group. The probability level of $p<0.05$ was considered the cut-off value for significance.

\section{Results}

3.1. Genomic DNA Extraction. The extracted genomic DNA was run on $1.5 \%$ agarose gel; then, the DNA bands were confirmed and visualized by Gel $\operatorname{Doc}^{\mathrm{TM}}$ and ChemiDoc ${ }^{\mathrm{TM}}$ Systems, Bio-Rad, USA. Gel electrophoresis of genomic DNA was done to confirm the presence of human genomic DNA in each sample.
3.2. NAT2 Amplification. This electrophoresis gel photograph shows the amplified fragment of the NAT2 gene. The band of $1150 \mathrm{bp}$ was confirmed by using DNA ladder as shown in Figure 1.

3.3. NAT2 Sequence Analysis. The commonly known seven SNPs detected in our study were $191 \mathrm{G}>\mathrm{A}, 282 \mathrm{C}>\mathrm{T}$, $341 \mathrm{~T}>\mathrm{C}, 481 \mathrm{C}>\mathrm{T}, 590 \mathrm{G}>\mathrm{A}, 803 \mathrm{~A}>\mathrm{G}$, and $857 \mathrm{G}>\mathrm{A}$ and their frequencies are represented in Table 1 . It has been noted that heterozygote SNPs are more common compared to homozygote SNPs (198 versus 99, respectively) as shown in Table 2.

3.4. Allele Frequency. The frequencies of these major NAT2 SNPs in all of the 96 individuals are represented in Table 1. This study found that $N A T 2 * 12,803 \mathrm{~A}>\mathrm{G}$, was the most frequent genetic variant (51\% of alleles) among Al-Ahsa population, whereas the genetic variant of $N A T 2 * 14,191 \mathrm{G}>\mathrm{A}$, was the least with allele frequency of only $1 \%$ of alleles. The allele not harboring any mutation was considered wild-type $N A T 2 * 4$, fast acetylator, which was present only in 2 cases of the 96 samples. So, the frequency of $N A T 2 * 4 / * 4$ was $2 \%$.

3.5. Genotype Frequency. The frequencies of NAT2 genotype obtained from all of subjects are publicized in Table 2. The most frequent observed heterozygote was NAT2*13 $282 \mathrm{C}>\mathrm{T}(43.7 \%)$ followed by $N A T 2 * 5341 \mathrm{~T}>\mathrm{C}(39.5 \%)$ and $N A T 2 * 12803 \mathrm{~A}>\mathrm{G}(39.5 \%)$ among Al-Ahsa volunteers. The lowest frequency of observed heterozygote genotypes was 
TABLE 2: The NAT2 gene representing different genotype frequencies among Al-Ahsa population $(n=96)$.

\begin{tabular}{lccc}
\hline NAT2 allele & $\begin{array}{c}\text { Wild type } \\
\text { Frequency (proportion, 95\% CI) }\end{array}$ & $\begin{array}{c}\text { Heterozygote } \\
\text { Frequency (proportion, 95\% CI) }\end{array}$ & $\begin{array}{c}\text { Homozygote } \\
\text { Frequency (proportion, 95\% CI) }\end{array}$ \\
\hline NAT2 $11(481 \mathrm{C}>\mathrm{T})$ & C/C: $39(0.406,0.308-0.504)$ & C/T: $35(0.365,0.268-0.461)$ & T/T: 22(0.229, 0.145-0.313) \\
NAT2 $12(803 \mathrm{~A}>\mathrm{G})$ & A/A: $28(0.292,0.201-0.383)$ & A/G: $38(0.396,0.298-0.494)$ & G/G: 30 $(0.313,0.22-0.405)$ \\
NAT2 $^{*} 13(282 \mathrm{C}>\mathrm{T})$ & C/C: $41(0.427,0.328-0.526)$ & C/T: $42(0.438,0.338-0.537)$ & T/T: $13(0.135,0.067-0.204)$ \\
NAT2 $^{*} 5(341 \mathrm{~T}>\mathrm{C})$ & T/T: $36(0.375,0.278-0.472)$ & T/C: $38(0.396,0.298-0.494)$ & C/C: 22(0.229, $0.145-0.313)$ \\
NAT2 $^{*} 6(590 \mathrm{G}>\mathrm{A})$ & G/G: $52(0.542,0.442-0.641)$ & G/A: $32(0.333,0.239-0.428)$ & A/A: $12(0.125,0.059-0.191)$ \\
NAT2 $7(857 \mathrm{G}>\mathrm{A})$ & G/G: $85(0.885,0.822-0.949)$ & G/A: $11(0.115,0.051-0.178)$ & A/A: $0(0.00)$ \\
NAT2 $^{*} 14(191 \mathrm{G}>\mathrm{A})$ & G/G: $94(0.979,0.951-1.008)$ & G/A: $2(0.021,-0.0077-0.049)$ & A/A: $0(0.00)$ \\
\hline
\end{tabular}

$N A T 2 * 14191 \mathrm{G}>\mathrm{A}$ with a frequency of $2 \%$. In homozygote, the $N A T 2 * 12803 \mathrm{~A}>\mathrm{G}(31.2 \%)$ genotype was the most common one but the lowest homozygote among them was NAT2 $* 6590 \mathrm{G}>\mathrm{A}(12.5 \%)$, and there was no any homozygote genotypes of $N A T 2 * 7587 \mathrm{G}>\mathrm{A}$ and $N A T 2 * 14191 \mathrm{G}>\mathrm{A}$ in the present study.

The Hardy-Weinberg equilibrium test was done by using the chi-squared $\left(\chi^{2}\right)$ test. Five of the seven most common SNPs were within Hardy-Weinberg equilibrium while two of them are not shown in Table 3.

3.6. Linkage Disequilibrium. The linkage disequilibrium (LD) analysis was done using Haploview 4.2 software to indicate the heterogeneity of Al-Ahsa population and the possibility of recombination among their NAT2 SNPs. As shown in Figure 2, the seven NAT2 variants, $191 \mathrm{G}>\mathrm{A}, 282 \mathrm{C}>\mathrm{T}$, $341 \mathrm{~T}>\mathrm{C}, 481 \mathrm{C}>\mathrm{T}, 590 \mathrm{G}>\mathrm{A}, 803 \mathrm{~A}>\mathrm{G}$, and $857 \mathrm{G}>\mathrm{A}$ were applied to Haploview software. The LD for each pair of genetic variants was measured using $\left|D^{\prime}\right|$ and correlation coefficient $\left(r^{2}>0.8\right)$. A haplotype block was found in the following SNP positions $282 \mathrm{C}>\mathrm{T}, 341 \mathrm{~T}>\mathrm{C}, 481 \mathrm{C}>\mathrm{T}, 590 \mathrm{G}>\mathrm{A}$, and $803 \mathrm{~A}>\mathrm{G}$ in $\mathrm{Al}$-Ahsa population samples which is identified as strong LD as represented in Figure 2.

3.7. Acetylation Phenotype. According to the different NAT2 polymorphisms, it will characterize the phenotypes into three different acetylators known as slow, intermediate, and fast [4], hence the predicted phenotypes in our study based on the various NAT2 haplotypes (Table 4). Consequently, our study revealed that the genotype frequency (predicted phenotype) of fast, intermediate, and slow acetylators was $5.21 \%$, 34.38\%, and $60.42 \%$, respectively (Table 4 ).

Many researchers who published work on NAT2, subdivided NAT $2 *$ phenotypes into either "slow" or "rapid" acetylators which is described as a bimodal pattern. However, the third type of acetylators, namely, "intermediate" is also mentioned in most of the NAT2 studies which have been called as a trimodal pattern. Both of them, bi- and trimodal acetylation classifications, have been found in the literature $[10,11]$.

3.8. Haplotype Frequency. The detected haplotypes of the NAT2 gene in the current study among Al-Ahsa population have been resulted from various SNPs existing together in one individual as explained in the NAT database nomenclature official website. In the NAT2 gene, the predicted acetyla- tion phenotypes and hence the metabolic capacity of this enzyme is determined by the haplotype structure for each individual. In the current study, we found in total 20 different haplotypes in Al-Ahsa population as reported in Table 5.

The most frequent two haplotypes in the present study were $N A T 2 * 6 C$ and $N A T 2 * 5 A$ with a frequency of $25 \%$ and $22.9 \%$, respectively, followed by NAT2*5B and NAT2* $5 U$ with a frequency of $11.5 \%$ and $12.5 \%$ correspondingly. That indicates most of examined samples of Al-Ahsa population were slow acetylators according to the bimodal distribution pattern with a proportion of $94.8 \%$ of slow acetylator phenotype and $5.21 \%$ of fast acetylator phenotype.

Our genotyping results detected four novel haplotypes which are not published yet on the NAT database nomenclature official website (http://nat.mbg.duth.gr/human\%20nat2\% 20alleles_2013.htm). The present study applied an online software NAT2PRED program (http://nat2pred.rit.albany.edu/) in order to predict its acetylation phenotype. Thus, the predicted phenotype of these four new haplotypes is slow acetylators as shown in Table 5, and they represent $9.37 \%$ of the 96 samples. The new detected haplotypes were given an official symbol based on communication with the Chair of the NAT Committee as the following NAT2*5TB, NAT2*5AB, $N A T 2 * 5 Z A$, and $N A T 2 * 6 \mathrm{~W}$ (Table 5), also see the attached supplementary file (available here).

3.9. Comparative Assessment of Al-Ahsa NAT2 Allele Frequency with Other Populations. The NAT2 genetic polymorphisms among the different ethnic groups and countries are common. Our work differentiates the frequencies of NAT2 allele among Al-Ahsa population with other ethnicities as illustrated in Table 6.

\section{Discussion}

This study is the first to provide an extensive and comprehensive report on NAT2 genotyping among Al-Ahsa population, in which all common polymorphisms of this specific gene have been thoroughly studied.

Unrelated samples were collected almost from each local district of Al-Ahsa to sufficiently cover most of the region, which represents a good sample distribution. The sample size of this study was relatively small which is correlated to a limitation of this research. Historically, it is undoubtedly perceived that Al-Ahsa inhabitants in the Eastern Province of Saudi Arabia have settled down the city thousands of 
TABLE 3: Observed and expected (by using Hardy-Weinberg equilibrium) frequencies of homozygote heterozygote NAT2 alleles among Al-Ahsa population.

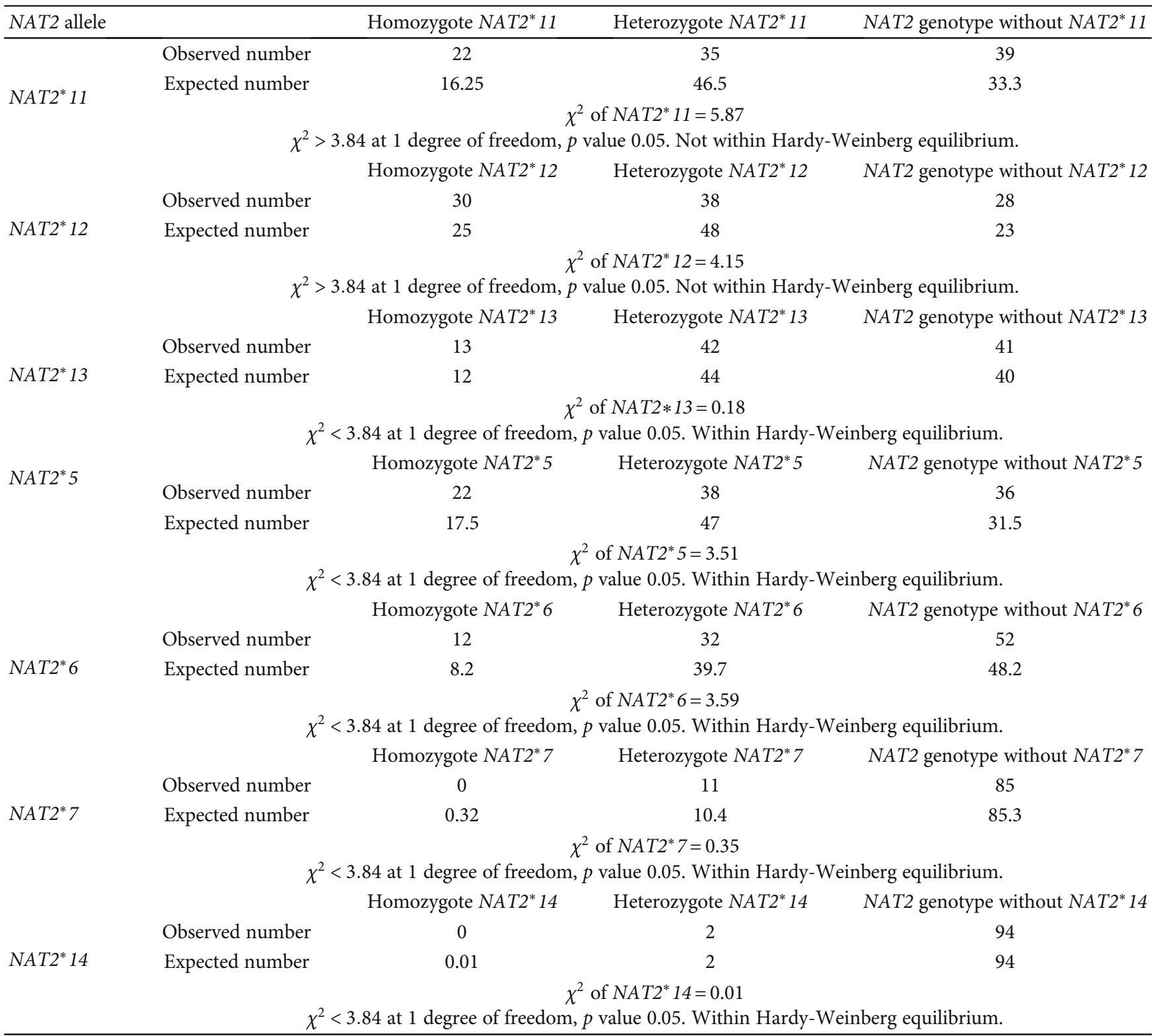

years ago and genetic problems are very common among them due to the apparent prevalence of endogamy. As a result, Al-Ahsa has a special demographic composition and unique population structure $[23,24]$. The acetylation rate has a great influence by the NAT2 gene due to the presence of various SNPs, and it may affect the drug metabolism and susceptibility to some diseases such as cancer. In the current work, the NAT2 gene whole exon 2 was sequenced from Al-Ahsa of Saudi population.

The frequency of slow NAT2 alleles in the current study $341 \mathrm{~T}>\mathrm{C} \quad(N A T 2 * 5), 590 \mathrm{G}>\mathrm{A} \quad(N A T 2 * 6)$, and $857 \mathrm{G}>\mathrm{A}$ $(N A T 2 * 7)$ were relatively similar to a previous Saudi study [12]. However, fast alleles 481C $>\mathrm{T}(N A T 2 * 11), 803 \mathrm{~A}>\mathrm{G}$ $(N A T 2 * 12)$, and $282 \mathrm{C}>\mathrm{T}(N A T 2 * 13)$ were not reported by $\mathrm{Bu}$ et al. compare to ours, which makes the current study more comprehensive.
The distribution of NAT2 polymorphisms in Al-Ahsa population appears to be relatively different from other ethnically related Arab populations, especially in the United Arab Emirates and Oman [14, 15]. The wild-type allele $(N A T 2 * 4)$ frequencies in Emiratis and Omanis are 0.13 and 0.18 , respectively, compared with our study $0.295[14,15]$. But it is slightly similar to Jordan and Egypt 0.23 and 0.22 , respectively. Besides, the frequency of $N A T 2 * 4$ allele in the current study is almost similar to Caucasians and different from South East Asians such as Japanese, Korean, and Chinese (Table 6).

The highest occurrence slow allele among Al-Ahsa population is $341 \mathrm{~T}>\mathrm{C}(N A T 2 * 5)$ with a frequency of $42.7 \%$, where the least common slow allele is $191 \mathrm{G}>\mathrm{A}(N A T 2 * 14)$ with a frequency of only $1 \%$. The later SNP (191G>A) was not examined in the previous Saudi study [12]. Conversely, the 


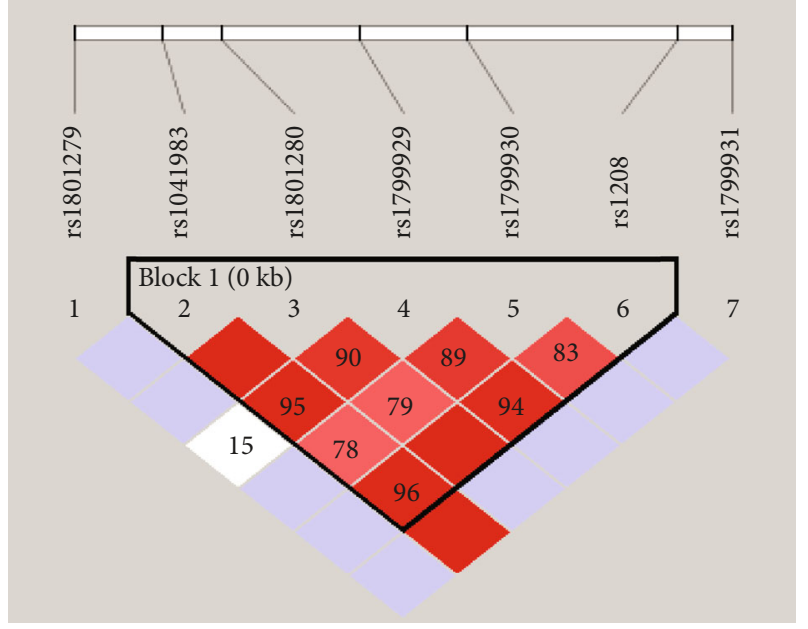

FIGURE 2: Linkage disequilibrium (LD) of NAT2 genetic variants found among Al-Ahsa population samples. The LD was carried out using Haploview software. The upper panel shows the location of 7 variants in the NAT2 gene, and the lower panel shows the output of Haploview. The red squares represent a strong LD, and the white square represents a weak LD. The blue square indicates that there is no LD.

TABle 4: Predicted acetylation phenotype among Al-Ahsa population $(n=96)$.

\begin{tabular}{lcc}
\hline $\begin{array}{l}\text { Acetylation } \\
\text { phenotype* }\end{array}$ & $\begin{array}{c}\text { Number of } \\
\text { samples }\end{array}$ & $\begin{array}{c}\text { Proportion } \\
\left(95 \% \mathrm{CI}^{* *}\right)\end{array}$ \\
\hline Fast & 5 & $0.05(0.01-0.09)$ \\
Intermediate & 33 & $0.34(0.25-0.44)$ \\
Slow & 58 & $0.61(0.51-0.70)$ \\
Total & 96 &
\end{tabular}

*The acetylation phenotype was determined depending on the trimodal distribution pattern based on online software http://nat2pred.rit.albany .edu/. ${ }^{* *} \mathrm{CI}$ : confidence interval.

(191G>A) SNP is absent in Omanis and European Caucasians, but it was reported among Emiratis with a significant frequency $(9.96 \%)$, and it was also found among Jordanians with less frequency $(0.7 \%)$. This polymorphism $(191 \mathrm{G}>\mathrm{A})$ is considered African specific with a frequency of $9 \%$ [25]. Moreover, this may explain its lower frequency in the present study and/or absent in others. Besides, the dissimilarity in the distribution of this SNP (191G>A) could be due to a lower degree of admixture in Al-Ahsa population. It has been reported that slow acetylators $N A T 2 * 5 B$ and $N A T 2 * 5 C$ are associated with a low risk of acute lymphoblastic leukemia (ALL) development. On the contrary, NAT2*5D is associated both with ALL and acute myeloblastic leukemia (AML) [26]. However, a Brazilian study demonstrated that $N A T 2 * 14, N A T 2 * 5 A$, and NAT2*5C are correlated with an increased susceptibility to ALL in a Brazilian population $[27,28]$. Recently, a research group reviewed the published literature on the correlation between NAT2 genetic variants and susceptibility to acute leukemia and it was reported that rs1801280 $(341 \mathrm{~T}>\mathrm{C}(N A T 2 * 5))$ contributes to the disease [29]. Having a high percentage of this $N A T 2 * 5 A$ polymor-
TABLE 5: NAT2 haplotypes among unrelated Al-Ahsa population $(n=96)$.

\begin{tabular}{|c|c|c|c|}
\hline Haplotype & Number & Frequency & Predicted phenotype \\
\hline $\mathrm{NAT}^{*} 6 \mathrm{C}$ & 24 & $25.00 \%$ & Slow \\
\hline$N A T 2 * 5 A$ & 22 & $22.92 \%$ & Slow \\
\hline$N A T 2 * 5 \mathrm{U}$ & 12 & $12.50 \%$ & Slow \\
\hline$N A T 2 * 5 B$ & 11 & $11.46 \%$ & Slow \\
\hline NAT2*5TB new & 7 & $7.29 \%$ & Slow \\
\hline$N A T 2 * 6 W$ new & 2 & $2.08 \%$ & Slow \\
\hline$N A T 2 * 5 G$ & 2 & $2.08 \%$ & Slow \\
\hline$N A T 2 * 5 R$ & 2 & $2.08 \%$ & Slow \\
\hline$N A T 2 * 6 A$ & 2 & $2.08 \%$ & Slow \\
\hline$N A T 2 * 4$ & 2 & $2.08 \%$ & Fast \\
\hline$N A T 2 * 5 A B$ new & 1 & $1.04 \%$ & Slow \\
\hline NAT2*5ZA new & 1 & $1.04 \%$ & Slow \\
\hline NAT2* $11 A$ & 1 & $1.04 \%$ & Fast \\
\hline NAT2* $12 A$ & 1 & $1.04 \%$ & Fast \\
\hline NAT2* $12 H$ & 1 & $1.04 \%$ & Fast \\
\hline$N A T 2^{*} 14 B$ & 1 & $1.04 \%$ & Slow \\
\hline$N A T 2 * 5 E$ & 1 & $1.04 \%$ & Slow \\
\hline NAT2*5TA & 1 & $1.04 \%$ & Slow \\
\hline$N A T 2^{*} 6 F$ & 1 & $1.04 \%$ & Slow \\
\hline$N A T 2^{*} 7 \mathrm{C}$ & 1 & $1.04 \%$ & Slow \\
\hline Total & 96 & $100 \%$ & \\
\hline
\end{tabular}

phism (23\%) among Al-Ahsa community may make them vulnerable to acute leukemia, taking into account that the Eastern region is the second in the prevalence of leukemia in the Kingdom after Riyadh [30].

The observed frequency of slow acetylator allele NAT2*6 among $\mathrm{Al}$-Ahsa community is $29 \%$ which is virtually consistent with other Arab countries (previous Saudi study 24\%, Jordan 30\%, Oman 30\%, and Egypt 26\%) and Caucasians $28 \%$. However, Emirates showed a lesser occurrence of $10 \%$ of that SNP (Table 6). The association between different NAT2 genotypes and risk for diabetes mellitus is controversial. Some groups showed no relationship between different NAT2 SNPs or phenotypes and risk for diabetes mellitus [31-33]. However, other groups showed a relationship between different NAT2 genotypes NAT2*6A, NAT2*5A, $N A T 2 * 14 A$, and $N A T 2 * 7 B$ and risk for diabetes mellitus $[34,35]$. The contemporary study revealed that the frequency of NAT2*7 (5.7\%) was in line with Arab and Caucasians (Oman 4\%, Jordan 3\%, Egypt 3\%, and Caucasians 2\%), except for Emirates and Asians which have a slightly more frequent $N A T 2 * 7$ allele $27 \%$ and $13 \%$, respectively, (Table 6).

It has been abstracted that the fast acetylators are connected with colorectal cancer especially with people who consume huge amounts of well-done meat that considered a predominant source of the carcinogenic heterocyclic amines which metabolized by NAT2 [36]. In addition, one thousands annually of Saudi patients out of 3 million people worldwide die of tuberculosis (TB) [37]. The infection rate of TB in the KSA remains high. This is reflected by the latest prevalence study which showed that TB range from $8.5 \%$ in 
TABLE 6: Distribution of NAT2 alleles among Al-Ahsa population compared with various.human population (no.of alleles = 192).

\begin{tabular}{|c|c|c|c|c|c|c|c|c|}
\hline Population & No. alleles & $N A T 2 * 4$ & $N A T 2 * 5$ & $N A T 2 * 6$ & $N A T 2 * 7$ & $N A T 2 * 11$ & $N A T 2^{*} 12$ & $N A T 2 * 13$ \\
\hline Saudi (current study) & 192 & 0.295 & 0.427 & 0.292 & 0.057 & 0.411 & 0.510 & 0.354 \\
\hline Saudi [12] & 974 & 0.27 & 0.47 & 0.24 & 0.02 & & & \\
\hline Emiratis [13] & 212 & 0.18 & 0.54 & 0.20 & 0.04 & & & \\
\hline Emiratis [14] & 1110 & 0.13 & 0.364 & 0.129 & 0.27 & & & \\
\hline Omanis [15] & 254 & 0.18 & 0.424 & 0.30 & 0.04 & 0 & 0.10 & 0 \\
\hline Jordan [16] & 136 & 0.23 & 0.485 & 0.30 & & 0.40 & 0.30 & 0.30 \\
\hline Egyptian [17] & 400 & 0.22 & 0.497 & 0.26 & 0.03 & & & \\
\hline Sudan $[18]$ & 254 & 0.087 & 0.473 & 0.28 & 0.03 & 0 & 0.08 & 0.001 \\
\hline Indians [19] & 188 & 0.10 & 0.346 & 0.20 & 0.05 & 0 & 0.30 & 0 \\
\hline Japan [20] & 346 & 0.66 & 0.01 & 0.20 & 0.13 & 0 & 0 & 0 \\
\hline Chinese & 560 & 0.60 & 0.136 & 0.40 & 0.43 & 0.1 & 0 & 0.6 \\
\hline Korean [21] & 2000 & 0.66 & 0.016 & 0.20 & 0.11 & 0 & 0 & 0 \\
\hline German [22] & 1688 & 0.22 & 0.46 & 0.27 & 0.013 & 0 & 0 & 0.15 \\
\hline
\end{tabular}

the central region (Riyadh) to as high as $23.1 \%$ in Hail for locals and as high as $38 \%$ for non-Saudis in the Makkah region [38]. The most effective drug used for treatment of $\mathrm{TB}$ is isoniazid (INH) which is mainly metabolized by NAT2 enzyme. The incidence of ADRs caused by INH is very high among slow acetylators, as Al-Ahsa population is mainly slow acetylators (60.42\%) (Table 6). Therefore, the dosage regimen of INH should be adjusted in TB patients carrying slow genotypes to prevent drug-induced liver injuries (DILI) and to decrease the cost of managing adverse events [39]. NAT2 genotypes are useful new biomarkers for predicting anti-TB DILI for TB patients. Accordingly, DNA-based diagnosis of TB patients before initiating treatment with anti-TB drugs may prove useful in achieving optimal treatment of individual TB patients.

In the present study, NAT2*5 prevalence is $42.7 \%$ and the NAT2*5 genotypes include (TT $37.5 \%$, TC $39.6 \%$, and CC $22.9 \%$ ). Association between NAT2*5 and anemia in ovarian cancer patients under the treatment of cisplatin was reported. Strictly, anemia was more prevalent among patients with the heterozygous TC genotype $(78.4 \%)$ compared with the homozygous wild-type [40]. Occasionally, heterozygous TC genotype, in our study, showed a $39.6 \%$ which reflects a high percentage of such SNP. So, it is recommended to do a NAT2 genotyping test before cisplatin treatment to reduce its ADRs.

Endometriosis is defined as the growth of endometrium in the peritoneal cavity, outside the uterine or myometrium [41]. It frequently occur among women, at the rate of $6-10 \%$ [42]. The prevalence of endometriosis was found to be $11-14.3 \%$ in Saudi women [43]. The relationship between endometriosis and polymorphisms in NAT2 gene was investigated in a UK population. The researchers reported that male controls account for $32 \%$ and unaffected women $33 \%$ consecutively. Therefore, they were found to be slow acetylators than the rate of $57 \%$ for women with stage III-IV endometriosis [42]. Interestingly, Fayez et al. found that the heterozygote genotype $590 \mathrm{G}$ A $(>N A T 2 * 6)$ SNP may be linked with vulnerability to endometriosis and the homozygote genotype 590 AA allele may have a protective role in development of endometriosis in Iranian women [44]. Our study showed that the distribution of heterozygote genotype $590 \mathrm{G}>\mathrm{A}(\mathrm{NAT} 2 * 6)$ was found to be $33 \%$ of Al-Ahsa population. This high percentage of prevalence genotype among Al-Ahsa population might make them susceptible to be affected by endometriosis.

\section{Conclusions}

Our study revealed the high prevalence of slow acetylators of NAT2 $(60.42 \%)$ in the studied samples among Al-Ahsa population which might alter drug's efficacy and vulnerability to some diseases, like cancer. The result of this study will be helpful to limit the unwanted side effects of some medications and maximization of its benefits to the patients. Moreover, we found four novel haplotypes which were given a new official NAT symbols $N A T 2 * 5 \mathrm{~TB}, N A T 2 * 5 A B$, $N A T 2 * 5 Z A$, and $N A T 2 * 6 \mathrm{~W}$ by the Chair of the international NAT Committee.

\section{Data Availability}

All data are within the manuscript.

\section{Ethical Approval}

This work was approved by the Research Ethics Committee (REC) at King Faisal University (REC REF NUMBER: KFU-REC/2017-11-02) and clinical ethics at King Fahad Hospital, Hufof (KFHH RCA NO: 07/09/39). A permission was taken before sampling and applied the rules of clinical ethics at King Fahad Hospital, Hufof (KFHH RCA NO: 07/09/39).

\section{Conflicts of Interest}

The authors declare no conflict of interest. 


\section{Authors' Contributions}

MA and AA designed the study; MA performed research; MA, AA, and MK analyzed data; MA, AA, and SA contributed new methods or models; MA, MK, SA, and AA revised the manuscript; and MA, MK, SA and AA wrote the paper.

\section{Acknowledgments}

The authors acknowledge the Deanship of Scientific Research at King Faisal University for the financial support under Nashir Track (Grant No. 186303). This work was funded by Deanship of Scientific Research (King Faisal University) under master students projects track (project no. 172034).

\section{Supplementary Materials}

Supplementary Table: NAT2 haplotypes among Al-Ahsa population. (Supplementary Materials)

\section{References}

[1] P. I. Mackenzie, A. A. Somogyi, and J. O. Miners, "Advances in drug metabolism and pharmacogenetics research in Australia," Pharmacological Research, vol. 116, pp. 7-19, 2017.

[2] A. Husain, X. Zhang, M. A. Doll, J. C. States, D. F. Barker, and D. W. Hein, "Identification of N-acetyltransferase 2 (NAT2) transcription start sites and quantitation of NAT2-specific mRNA in human tissues," Drug Metabolism and Disposition, vol. 35, no. 5, pp. 721-727, 2007.

[3] S. Boukouvala and G. Fakis, "ArylamineN-Acetyltransferases: what we learn from genes and genomes," Drug Metabolism Reviews, vol. 37, no. 3, pp. 511-564, 2008.

[4] E. Garcia-Martin, "Interethnic and intraethnic variability of NAT2 single nucleotide polymorphisms," Current Drug Metabolism, vol. 9, no. 6, pp. 487-497, 2008.

[5] N. A. Kshirsagar, S. M. Pohujani, M. R. Takle, V. N. Acharya, and R. S. Satoskar, "Sulphadimidine acetylation status in Gujarati and Marathi population," Journal of Postgraduate Medicine, vol. 33, no. 3, pp. 128-133, 1987.

[6] Y. Zang, M. A. Doll, S. Zhao, J. C. States, and D. W. Hein, "Functional characterization of single-nucleotide polymorphisms and haplotypes of human N-acetyltransferase 2," Carcinogenesis, vol. 28, no. 8, pp. 1665-1671, 2007.

[7] D. W. Hein, "N-acetyltransferase 2 genetic polymorphism: effects of carcinogen and haplotype on urinary bladder cancer risk," Oncogene, vol. 25, no. 11, pp. 1649-1658, 2006.

[8] D. W. Hein, M. A. Doll, A. J. Fretland et al., "Molecular genetics and epidemiology of the NAT1 and NAT2 acetylation polymorphisms," Cancer Epidemiology, Biomarkers \& Prevention, vol. 9, no. 1, pp. 29-42, 2000.

[9] P. Baldi, S. Brunak, Y. Chauvin, C. A. F. Andersen, and H. Nielsen, "Assessing the accuracy of prediction algorithms for classification: an overview," Bioinformatics, vol. 16, no. 5, pp. 412-424, 2000.

[10] W. WEBER, "Populations and genetic polymorphisms," Molecular Diagnosis, vol. 4, no. 4, pp. 299-307, 1999.

[11] M. Ohno, I. Yamaguchi, I. Yamamoto et al., "Slow Nacetyltransferase 2 genotype affects the incidence of isoniazid and rifampicin-induced hepatotoxicity," The International
Journal of Tuberculosis and Lung Disease, vol. 4, no. 3, pp. 256-261, 2000.

[12] R. Bu, M. I. Gutiérrez, M. al-Rasheed, A. Belgaumi, and K. Bhatia, "Variable drug metabolism genes in Arab population," The Pharmacogenomics Journal, vol. 4, no. 4, pp. 260 266, 2004.

[13] N. M. Woolhouse, M. M. Qureshi, S. M. A. Bastaki, M. Patel, Y. Abdulrazzaq, and R. A. L. Bayoumi, "Polymorphic Nacetyltransferase (NAT2) genotyping of Emiratis," Pharmacogenetics, vol. 7, no. 1, pp. 73-82, 1997.

[14] M. M. Al-Ahmad, N. Amir, S. Dhanasekaran et al., "Studies on $\mathrm{N}$-Acetyltransferase (NAT2) genotype relationships in Emiratis: confirmation of the existence of phenotype variation among slow acetylators," Annals of Human Genetics, vol. 81, no. 5, pp. 190-196, 2017.

[15] M. O. Tanira, M. Simsek, K. al Balushi, K. al Lawatia, H. al Barawani, and R. A. Bayoumi, "Distribution of arylamine $\mathrm{N}$-acetyltransferase 2 (nat 2) genotypes among Omanis," Journal for scientific research. Medical sciences/Sultan Qaboos University, vol. 5, no. 1-2, pp. 9-14, 2003.

[16] Y. B. Jarrar, A. A. Balasmeh, and W. Jarrar, "Sequence analysis of the N-acetyltransferase 2 gene (NAT2) among Jordanian volunteers," Libyan Journal of Medicine, vol. 13, no. 1, p. 1408381, 2017.

[17] S. I. Hamdy, M. Hiratsuka, K. Narahara et al., "Genotype and allele frequencies of TPMT, NAT2, GST, SULT1A1 and MDR-1 in the Egyptian population," British Journal of Clinical Pharmacology, vol. 55, no. 6, pp. 560-569, 2003.

[18] S. Al-Yahyaee, U. Gaffar, M. M. Al-Ameri et al., "N-acetyltransferase polymorphism among northern Sudanese," Human Biology, vol. 79, no. 4, pp. 445-452, 2007.

[19] C. Neelam and P. Harish, "Variants of NAT2 polymorphisms: intra and inter-ethnic differences," African Journal of Biotechnology, vol. 13, no. 51, pp. 4639-4646, 2014.

[20] T. Hamasaki, H. Inatomi, T. Katoh et al., "N-acetyltransferase2 gene polymorphism as a possible biomarker for prostate cancer in Japanese men," International Journal of Urology, vol. 10, no. 3, pp. 167-173, 2003.

[21] H. J. Lin, C. Y. Han, B. K. Lin, and S. Hardy, "Ethnic distribution of slow acetylator mutations in the polymorphic $\mathrm{N}$-acetyltransferase (NAT2) gene," Pharmacogenetics, vol. 4, no. 3, pp. 125-134, 1994.

[22] I. Cascorbi, N. Drakoulis, J. Brockmöller, A. Maurer, K. Sperling, and I. Roots, "Arylamine N-acetyltransferase (NAT2) mutations and their allelic linkage in unrelated Caucasian individuals: correlation with phenotypic activity," American Journal of Human Genetics, vol. 57, no. 3, pp. 581-592, 1995.

[23] A. Bittles, "Consanguinity and its relevance to clinical genetics," Clinical Genetics, vol. 60, no. 2, pp. 89-98, 2001.

[24] M. I. El-Mouzan, A. A. Al-Salloum, A. S. Al-Herbish, M. M. Qurachi, and A. A. Al-Omar, "Regional variations in the prevalence of consanguinity in Saudi Arabia," Saudi Medical Journal, vol. 28, no. 12, pp. 1881-1884, 2007.

[25] D. A. Bell, J. A. Taylor, M. A. Butler et al., "Genotype/phenotype discordance for human arylamine $\mathrm{N}$-acetyltransferase (NAT2) reveals a new slow-acetylator allele common in African-Americans," Carcinogenesis, vol. 14, no. 8, pp. 16891692, 1993.

[26] A. M. Kamel, G. T. A. Ebid, and H. S. Moussa, "N-Acetyltransferase 2 (NAT2) polymorphism as a risk modifier of 
susceptibility to pediatric acute lymphoblastic leukemia," Tumour Biology, vol. 36, no. 8, pp. 6341-6348, 2015.

[27] C. W. Zanrosso, M. Emerenciano, B. A. . A. Goncalves, A. Faro, S. Koifman, and M. S. Pombo-de-Oliveira, "Nacetyltransferase 2 polymorphisms and susceptibility to infant leukemia with maternal exposure to dipyrone during pregnancy," Cancer Epidemiology, Biomarkers \& Prevention, vol. 19, no. 12, pp. 3037-3043, 2010.

[28] C. W. Zanrosso, M. Emerenciano, A. Faro, B. A. de Aguiar Gonçalves, M. B. Mansur, and M. S. Pombo-de-Oliveira, "Genetic variability in $\mathrm{N}$-acetyltransferase 2 gene determines susceptibility to childhood lymphoid or myeloid leukemia in Brazil," Leukemia \& Lymphoma, vol. 53, no. 2, pp. 323-327, 2011.

[29] X. Zhu, Y. Liu, G. Chen et al., "Association between NAT2 polymorphisms and acute leukemia risk: a meta-analysis," Medicine (Baltimore), vol. 98, no. 12, article e14942, 2019.

[30] G. A. Ibrahim, I. H. Issam, S. A. Mohamed, A. D. Ahlam, and A. E. S. Mohamed, "The incidence of leukemia in Saudi Arabia: Descriptive epidemiological analysis of data from the Saudi Cancer Registry (2001-2008)," Saudi Medizinhistorisches Journal, vol. 35, no. 7, pp. 674-683, 2014.

[31] S. Neugebauer, T. Baba, T. Watanabe, T. Ishizaki, and K. Kurokawa, "The N-acetyltransferase (NAT) gene: an early risk marker for diabetic nephropathy in Japanese type 2 diabetic patients?," Diabetic Medicine, vol. 11, no. 8, pp. 783-788, 1994.

[32] J. A. Agúndez, J. G. Menaya, R. Tejeda, F. Lago, M. Chávez, and J. Benítez, "Genetic analysis of the NAT2 and CYP2D6 polymorphisms in white patients with non-insulindependent diabetes mellitus," Pharmacogenetics, vol. 6, no. 5, pp. 465-472, 1996.

[33] R. A. Hegele, K. Kwan, S. B. Harris, A. J. G. Hanley, B. Zinman, and H. Cao, "NAT2 polymorphism associated with plasma glucose concentration in Canadian Oji-Cree," Pharmacogenetics, vol. 10, no. 3, pp. 233-238, 2000.

[34] S. Yalin, R. Hatungil, L. Tamer et al., "N-acetyltransferase 2 polymorphism in patients with diabetes mellitus," Cell Biochemistry and Function, vol. 25, no. 4, pp. 407-411, 2007.

[35] S. Semiz, T. Dujic, B. Ostanek et al., "Association of NAT2 polymorphisms with type 2 diabetes in a population from Bosnia and Herzegovina," Archives of Medical Research, vol. 42, no. 4, pp. 311-317, 2011.

[36] K. F. Ilett, B. M. David, P. Detchon, W. M. Castleden, and R. Kwa, "Acetylation phenotype in colorectal carcinoma," Cancer Research, vol. 47, no. 5, pp. 1466-1469, 1987.

[37] S. A. Al-Hajoj, "Tuberculosis in Saudi Arabia: can we change the way we deal with the disease?," Journal of Infection and Public Health, vol. 3, no. 1, pp. 17-24, 2010.

[38] A. Watban, A. Salamah, and M. Faki, "Prevalence of suspected tuberculosis in the Kingdom of Saudi Arabia according to conventional and molecular methods," Journal of Family and Community Medicine, vol. 21, no. 3, pp. 182-185, 2014.

[39] J. Azuma, Pharmacogenetics-based tuberculosis therapy research group, M. Ohno et al., "NAT2 genotype guided regimen reduces isoniazid-induced liver injury and early treatment failure in the 6-month four-drug standard treatment of tuberculosis: a randomized controlled trial for pharmacogenetics-based therapy," European Journal of Clinical Pharmacology, vol. 69, no. 5, pp. 1091-1101, 2013.
[40] A. V. Khrunin, D. V. Khokhrin, A. A. Moisseev, V. A. Gorbunova, and S. A. Limborska, "Pharmacogenomic assessment of cisplatin-based chemotherapy outcomes in ovarian cancer," Pharmacogenomics, vol. 15, no. 3, pp. 329-337, 2014.

[41] P. Bellelis, S. Podgaec, and M. M. Brão, "Environmental factors and endometriosis," Revista da Associação Médica Brasileira, vol. 57, no. 4, pp. 448-452, 2011.

[42] L. C. Giudice and L. C. Kao, "Endometriosis," Lancet, vol. 364, no. 9447, pp. 1789-1799, 2004.

[43] A. A. Rouzi, N. Sahly, S. Kafy, D. Sawan, and H. Abduljabbar, "Prevalence of endometriosis at a university hospital in Jeddah, Saudi Arabia," Clinical and Experimental Obstetrics and Gynecology, vol. 42, no. 6, pp. 785-786, 2015.

[44] D. Fayez, K. Saliminejad, S. Irani, K. Kamali, T. Memariani, and H. R. Khorram Khorshid, "Arylamine N-acetyltransferase 2 polymorphisms and the risk of endometriosis," Avicenna Journal of Medical Biotechnology, vol. 10, no. 3, pp. 163-167, 2018. 